\title{
An Element Weakly Primary to Another Element
}

\author{
C. S. Manjarekar' ${ }^{1}$ and U. N. Kandale ${ }^{2}$ \\ ${ }^{1}$ Department of Mathematics, Shivaji University, Kolhapur, India \\ ${ }^{2}$ Department of Mathematics, Sharad Institute of Technology, College of Engineering, Yadrav, Ichalkaranji, India
}

Correspondence should be addressed to U. N. Kandale; ujwalabiraje@gmail.com

Received 3 August 2013; Accepted 25 August 2013

Academic Editors: Z.-Y. Li, Y. Ouyang, and W. Zhu

Copyright (C) 2013 C. S. Manjarekar and U. N. Kandale. This is an open access article distributed under the Creative Commons Attribution License, which permits unrestricted use, distribution, and reproduction in any medium, provided the original work is properly cited.

We introduce the concept "An element weakly primary to another element" and using this concept we have generalized some result proved by Manjarekar and Chavan (2004). It is shown that if $\left\{b_{\alpha}\right\}$ is a family of elements weakly primary to $a$ in $L$, then $\bigvee_{\alpha} b_{\alpha}$ is weakly primary to $a$.

\section{Introduction}

Multiplicative lattice is a complete lattice provided with commutative, associative, and join distributive multiplication for which the largest element 1 acts as a multiplicative identity. A proper element $p$ of $L$ is called prime element if $a b \leqslant p \Rightarrow$ $a \leqslant p$ or $b \leqslant p$ for $a, b \in L$ and is called primary element if $a b \leqslant p$ implies $a \leqslant p$ or $b^{n} \leqslant p$ for some $n \in Z_{+}$. An element $a$ of $L$ is called compact if $a \leqslant \vee X$, and $X \subseteq L$ implies the existence of finite number of elements $X_{1}, X_{2}, X_{3}, \ldots, X_{n}$ of $L$ such that $a \leqslant X_{1} \vee X_{2} \vee X_{3} \vee \cdots \vee X_{n}$. Throughout this paper, $L$ denotes compactly generated multiplicative lattice with 1 compact and every finite product of compact elements is compact. Let $L_{*}$ be the set of all compact elements in $L$. Also, $(a: b)$ is the greatest element $c$ in $L$ such that $c b \leqslant a$. An element $a \in L$ is join principal if $x \vee(y: a)=(x a \vee y): a$ and meet principle if $x \wedge y a=((x: a) \wedge y) a$, for all $x, y \in L$.

An element is principle if it is both join and meet principle. For $a \in L, \sqrt{a}=\vee\left\{x \in L_{*} \mid x^{n} \leqslant a\right.$ for some $n \in$ $\left.Z_{+}\right\}$. An element $a \in L$ is called semiprimary if $\sqrt{a}$ is primary element. $L$ is said to satisfy the condition $(*)$ if every semiprimary element is primary element.

An element $a \in L$ is said to be strong join principle element if $a$ is compact and join principle. An element $a \in L$ is $p$-primary if $a$ is primary and $\sqrt{a}=p$ and $a \in L$ is semiprime if $\sqrt{a}=a$. An element $a$ of $L$ is called zero divisor if $\exists 0 \neq b \in L$ such that $a b=0$, and if $L$ has no zero divisor then $L$ will be called lattice domain or simply a domain. $L_{*}$ denotes the set of compact elements of $L$.

The concept of weakly prime element is studied by Çallialp et al. [1]. The concept of weakly primary element is introduced by Sachin and Vilas [2]. For other definitions and simple properties of multiplicative lattice, one can refer to Dilworth [3].

Definition 1. Weakly primary element is defined as follows.

An element $q \in L$ is said to be a weakly primary element if for $a, b \in L *, 0 \neq a b \leqslant q$ implies $a \leqslant q$ or $b^{n} \leqslant q$ for some $n \in Z_{+}$.

Example 2. Lattice of ideals of ring $R=\left\langle Z_{12},+, \cdot\right\rangle$ (see Figure 1).

In the lattice of Example 2, an element $a$ is weakly primary element. From Definition 1, it is clear that every weakly prime element is weakly primary element Converse need not be true. Since in Example 2, $a$ is weakly primary element but it is not weakly prime element. Further, if $q$ is a weakly primary element, then $\sqrt{q}$ is a weakly prime element. Because if for compact element $x$ and $y$ such that $0 \neq x y \leqslant \sqrt{q}$ then $x^{n} y^{n}=$ $(x y)^{n} \leqslant q$ for some $n \in Z_{+}$. As $q$ is a weakly primary element, either $x^{n} \leqslant q$ or $\left(y^{n}\right)^{m}=y^{n m} \leqslant q$ for some $n \in Z_{+}$. Consequently, $x \leqslant \sqrt{q}$ or $y \leqslant \sqrt{q}$. Thus, $\sqrt{q}$ is a weakly prime 


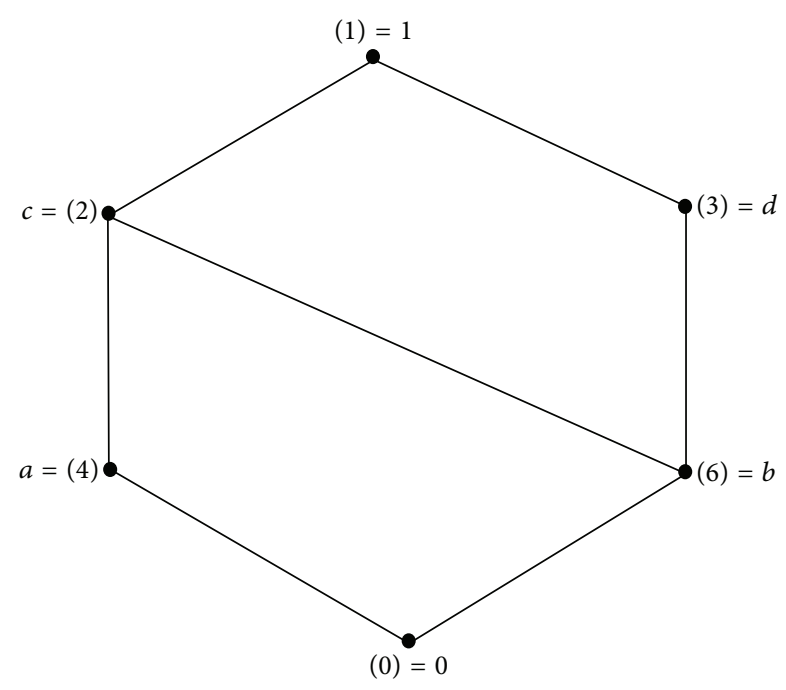

FIGURE 1

element. This implies that every weakly primary element is a weakly semiprimary element. It need not be true that $a$ is always weakly prime or $a$ is always weakly semiprimary. In Example 2, the least element 0 is not semiprimary as $\sqrt{0}=b$ is not a weakly prime element. The concept of "An element primeto another element" is introduced in [4]. An element $b \in L$ is primeto an element $a$ if for $x \in L, x b \leqslant a$ implies $x \leqslant a$.

Now, we define the following.

Definition 3. An element weakly prime to another element is defined as follows.

An element, $b \in L$, is called weakly prime to an element $a \in L$ if for any $x \in L, 0 \neq x b \leqslant a$ implies $x \leqslant a$.

In Example 2, the element $d$ is weakly prime to an element $c$, but $d$ is not weakly prime to any other element of $L$. This follows directly from the fact that an element $y$ is weakly prime to an element $x$ if and only if $x: y=x$.

\section{An Element Weakly Primary to Another Element}

Now we introduce the following main concept which is a generalization of the concept introduced by Manjarekar and Chavan [5].

Definition 4. An element $b$ is said to be weakly primary to another element $a$ in $L$ if for $x \in L_{*}, 0 \neq x b \leqslant a$ implies $x^{n} \leqslant a$ for some $n \in Z_{+}$.

In Example 2, the element $b$ is weakly primary to $a$, but note that $b$ is not weakly prime to $a$. This follows directly from Corollary 9, and note that $(a: b)=c=\sqrt{a}$ and $a<c$. Evidently, if $b$ is weakly prime to $a$, then $b$ is weakly primary to $a$ in $L$. Now if $p$ is a weakly prime element and $a \nless p$, then $a$ is weakly prime to $p$, and if $q$ is a weakly primary element and a compact element $a \nless q$, then $a$ is a weakly primary element to $q$.
Thus, from this, it is clear that elements weakly primary to another element exist in the lattice $L$. Since $L$ is compactly generated multiplicative lattice with 1 compact, weakly prime element and hence weakly primary element exists in $L$. Hereafter, $L$ will be a domain. We prove some interesting results including characterizations.

Theorem 5. No proper nonzero element is weakly prime or weakly primary to itself in $L$.

Proof. If $a$ is a proper nonzero element in $L$ and $a$ is weakly primary to $a$ itself, then $0 \neq a .1=a$ implies that $1=a$, a contradiction. Therefore, no proper nonzero element is weakly prime or weakly primary to itself in $L$.

Now we prove some characterizations of an element weakly primary to $a$.

Theorem 6. Let $a \in L$ be a semiprime element. Then $0 \neq b$ is weakly primary to $a$ if and only if $b$ is weakly prime to $a$.

Proof. Assume that $b$ is weakly primary to $a$ semiprime element $a$. Let $0 \neq x b \leqslant a$ for some $x \in L *$. Then $x^{n} \leqslant a$ for some $n \in Z_{+}$. Consequently, $x \leqslant \sqrt{a}$. As $a$ is semiprime, $x \leqslant a$. Thus, $b$ is weakly prime to $a$. The converse part is obvious.

Theorem 7. Let $L$ be a lattice domain. Let $a, b \in L$; then $0 \neq b$ is weakly primary to $a$ if and only if $(a: b) \leqslant \sqrt{a}$.

Proof. Assume that $0 \neq b$ is weakly primary to $a$. Let $0 \neq x \in$ $L_{*}$ such that $0 \neq x \leqslant(a: b)$; then, $0 \neq x b \leqslant a$. As $b$ is weakly primary to $a, x^{n} \leqslant a$ for some $n \in Z_{+}$. Hence, $x \leqslant \sqrt{a}$. This shows that $(a: b) \leqslant \sqrt{a}$.

Conversely, assume that $(a: b) \leqslant \sqrt{a}$. Let $0 \neq x b \leqslant a$ for some $x \in L *$. Then, we have $x \leqslant(a: b) \leqslant \sqrt{a}$. This implies that $x^{n} \leqslant a$ for some $n \in Z_{+}$. Thus, $b$ is weakly primary to $a$.

Theorem 8. Let $L$ be a lattice domain. Let $a, b \in L$; then $0 \neq b$ is weakly prime to $a$ if and only if $a=(a: b)$.

Proof. Assume that $0 \neq b$ is weakly prime to $a$ in $L$. Let $0 \neq x \in$ $L$ such that $x \leqslant(a: b)$. Then $0 \neq x b \leqslant a$. As $b$ is weakly prime to $a$, we have $x \leqslant a$. This shows that $(a: b) \leqslant a$. But $a \leqslant(a: b)$. Therefore, we get $a=(a: b)$.

Conversely, assume that $(a: b)=a$. Let, $0 \neq x b \leqslant a$ for $x \in L$. Then, we get $x \leqslant(a: b)=a$. Thus $b$ is weakly prime to $a$.

Corollary 9. Let $L$ be a lattice domain. Let, $a, b \in L$. Then, $0 \neq b$ is weakly primary to a but it is nonweakly prime to $a$ if and only if $a<(a: b) \leqslant \sqrt{a}$.

Proof. It follows from the fact that $a \leqslant(a: b)$ and from Theorems 7 and 8.

Corollary 10. Let $a, b \in L$. If $a$ is weakly semiprimary element and $b \nless \sqrt{a}$, then $0 \neq b$ is weakly primary to $a$. 
Proof. Assume that $a \in L$ is a weakly semiprimary element and $b \leqslant \sqrt{a}$. Let $x \in L *$ such that $0 \neq x b \leqslant a$. Then $0 \neq x b \leqslant$ $\sqrt{a}$. As $\sqrt{a}$ is a weakly prime element and $b \nless \sqrt{a}$, we have $x \leqslant \sqrt{a}$. This implies that $x^{n} \leqslant a$ for some $n \in Z_{+}$. Thus, $b$ is weakly primary to $a$.

Theorem 11. Let $L$ be a lattice domain. Let $a, b \in L$. Then, $0 \neq b$ is weakly prime to $a$ if and only if $b$ is weakly prime to $(a: x)$ for every $0 \neq x \in L$.

Proof. Assume that $b$ is weakly prime to $a$ in $L$. Let $0 \neq y b \leqslant$ $(a: x)$ for some $y \in L$. Then, $0 \neq x y b \leqslant a$. As $b$ is weakly prime to $a, x y \leqslant a$. Consequently, $y \leqslant(a: x)$. Thus, $b$ is weakly prime to $(a: x)$ for every $0 \neq x \in L$. The converse is obvious, since, if $b$ is weakly prime to $(a: x)$ for every $0 \neq x \in$ $L, b$ is weakly prime to $(a: 1)=a$.

Theorem 12. Let $a, b \in L$ and let $L$ be a lattice domain. If $b$ is weakly primary to $a$ and $a$ is a semiprime element in $L$, then $b$ is weakly primary to $(a: x)$ for every $x \in L$.

Proof. It follows from Theorems 6 and 11 .

Theorem 13. Let $a, b \in L$. Then $0 \neq b$ is weakly primary to $a$ in $L$ if and only if each $x \geqslant a$ is weakly primary to $a$.

Proof. Assume that $0 \neq b$ is weakly primary to $a$ in $L$. Let $x \geqslant b$ and $y x \leqslant a$ for some $y \in L *$. Then $0 \neq y b \leqslant a$. Therefore, by assumption, $y^{n} \leqslant a$ for some $n \in Z_{+}$.

This shows that each $x \geqslant b$ is weakly prime to $a$. The converse part is obvious.

Theorem 14. If $\left\{b_{\alpha}\right\}$ is a family of a elements weakly primary to $a$ in $L$, then $\bigvee_{\alpha} b_{\alpha}$ is weakly primary to $a$.

Proof. It follows from the fact that $b_{\alpha} \leqslant \bigvee_{\alpha} b_{\alpha}$ and from Theorem 13.

Theorem 15. Let $a, b \in L$. Then, $0 \neq b$ is nonweakly primary to $a$ if and only if each $x \leqslant b$ is nonweakly primary to $a$.

Proof. Assume that $b$ is nonweakly primary to $a$. Therefore, by Theorem 8 , we have $a: b \nless \sqrt{a}$. Let $x$ be an element of $L$ such that $x \leqslant b$. Then $(a: b) \leqslant(a: x)$. This shows that $(a: x) \nless \sqrt{a}$. Thus, again by Theorem 8 , each $x \leqslant b$ is nonweakly primary to $a$. laries.

This lemma leads us to the following two obvious corol-

Corollary 16. If $\left\{b_{\alpha}\right\}$ is a family of elements nonweakly primary to $a$ in $L$, then $\bigwedge_{\alpha} b_{\alpha}$ is nonweakly primary to $a$.

Corollary 17. If $\bigvee_{\alpha} b_{\alpha}$ is nonweakly primary to $a$ in $L$, then each $b_{\alpha}$ is nonweakly primary to $a$.

Theorem 18. If $y$ is compact and $0 \neq x y$ is nonweakly primary to $a$, then either $x$ is nonweakly primary to a or $y^{n}$ is nonweakly primary to a for some $n \in Z_{+}$.

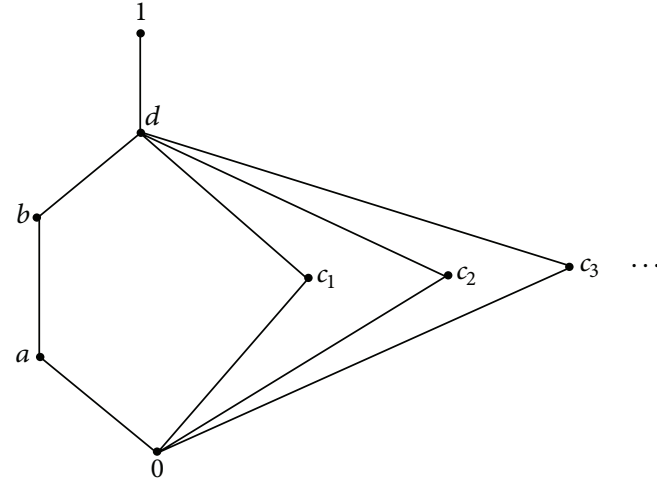

Figure 2

Definition 19. Completely meet semiprimary elements are defined as follows.

An element $a$ is said to be completely meet semiprimary element if $\sqrt{a}$ is a completely meet prime element.

Example 20. Every element $x<d$ is not a completely meet prime element. But note that $\sqrt{x}=d$. Thus, each $x<d$ is a completely meet semiprimary element (see Figure 2).

Result 1. In any multiplicative lattice $S$, we have $\bigwedge_{\alpha}\left(a: x_{\alpha}\right)=$ $a:\left(\bigvee_{\alpha} x_{\alpha}\right)$ for any $a, x_{\alpha} \in S$.

Theorem 21. Suppose that a is a completely meet semiprimary element of $L$ and let $\left\{b_{\alpha}\right\} \subseteq L$. If each $0 \neq b_{\alpha}$ is nonweakly primary to $a$ in $L$, then $\bigvee_{\alpha} b_{\alpha}$ is nonweakly primary to $a$.

Proof. By Result 1, we have $a:\left(\bigvee_{\alpha} b_{\alpha}\right)=\bigwedge_{\alpha}\left(a: b_{\alpha}\right)$. But each $0 \neq b_{\alpha}$ is nonweakly primary to $a$. Consequently, by Theorem 12 , each $\left(a: b_{\alpha}\right) \nless \sqrt{a}$. As $a$ is completely meet semiprimary element, $\bigwedge_{\alpha}\left(a: b_{\alpha}\right)=a:\left(\bigvee_{\alpha} b_{\alpha}\right)$ $\sqrt{a}$. Therefore, by Theorem $8, \bigvee_{\alpha} b_{\alpha}$ is nonweakly primary to a.

Now we construct a new element as follows. Define $q_{a}=$ $\vee\{x \in L \mid x$ is non weakly primary to $a\}$.

Theorem 22. If a is a completely meet semiprimary element, then $q_{a}$ is weakly primary element and is nonweakly primary to $a$.

Proof. By Theorem 21, it follows that $q_{a}$ is nonweakly primary element to $a$. Let $x$ and $y$ be compact elements such that, $0 \neq x y \leqslant q_{a}$. Then by Theorem $15, x y$ is nonweakly primary to $a$. Therefore, by Theorem 18, either $x$ is nonweakly primary to $a$ or $y^{n}$ is nonweakly primary to $a$ for some $n \in Z_{+}$. Thus, $x \leqslant q_{a}$ or $y^{n} \leqslant q_{a}$ for some $n \in Z_{+}$. This shows that $q_{a}$ is a weakly primary element.

\section{References}

[1] F. Çallialp, C. Jayram, and Ü. Tekir, "Weakly prime element in multilplicative lattices," Communication in Algebra, vol. 40, no. 8, pp. 2825-2840, 2012. 
[2] B. Sachin and K. Vilas, "On weakly primary element in multiplicative lattices," Communicated.

[3] R. P. Dilworth, "Abstarct commutative ideal theory," Pacific Journal of Mathematics, vol. 12, no. 2, pp. 481-498, 1962.

[4] F. Alarcon, D. D. Anderson, and C. Jayaram, "Some results on abstract commutative ideal theory," Periodica Mathematica Hungarica, vol. 30, no. 1, pp. 1-26, 1995.

[5] C. S. Manjarekar and N. S. Chavan, "An element primary to another element," The Journal of the Indian Mathematical Society, vol. 71, no. 1-4, pp. 55-60, 2004. 


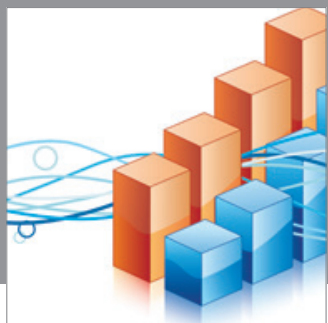

Advances in

Operations Research

mansans

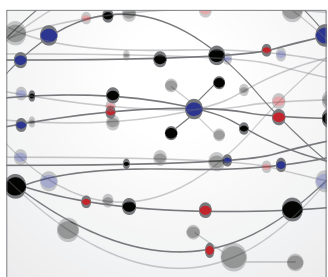

The Scientific World Journal
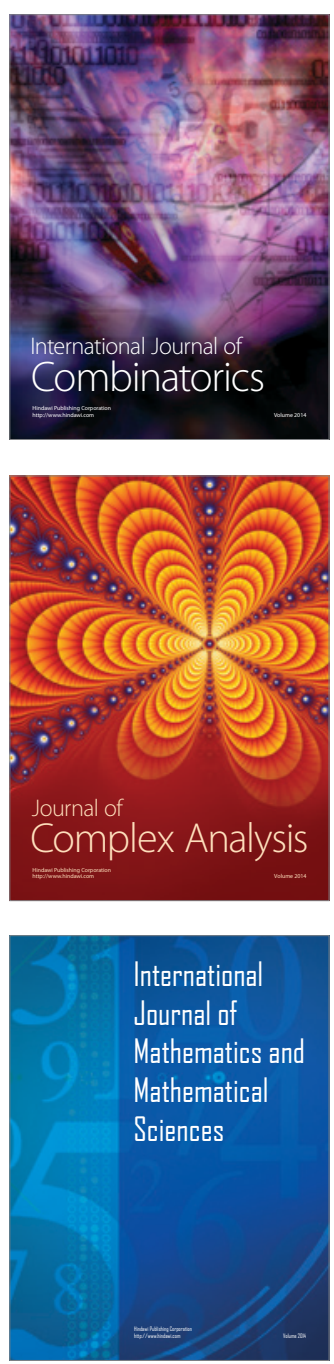
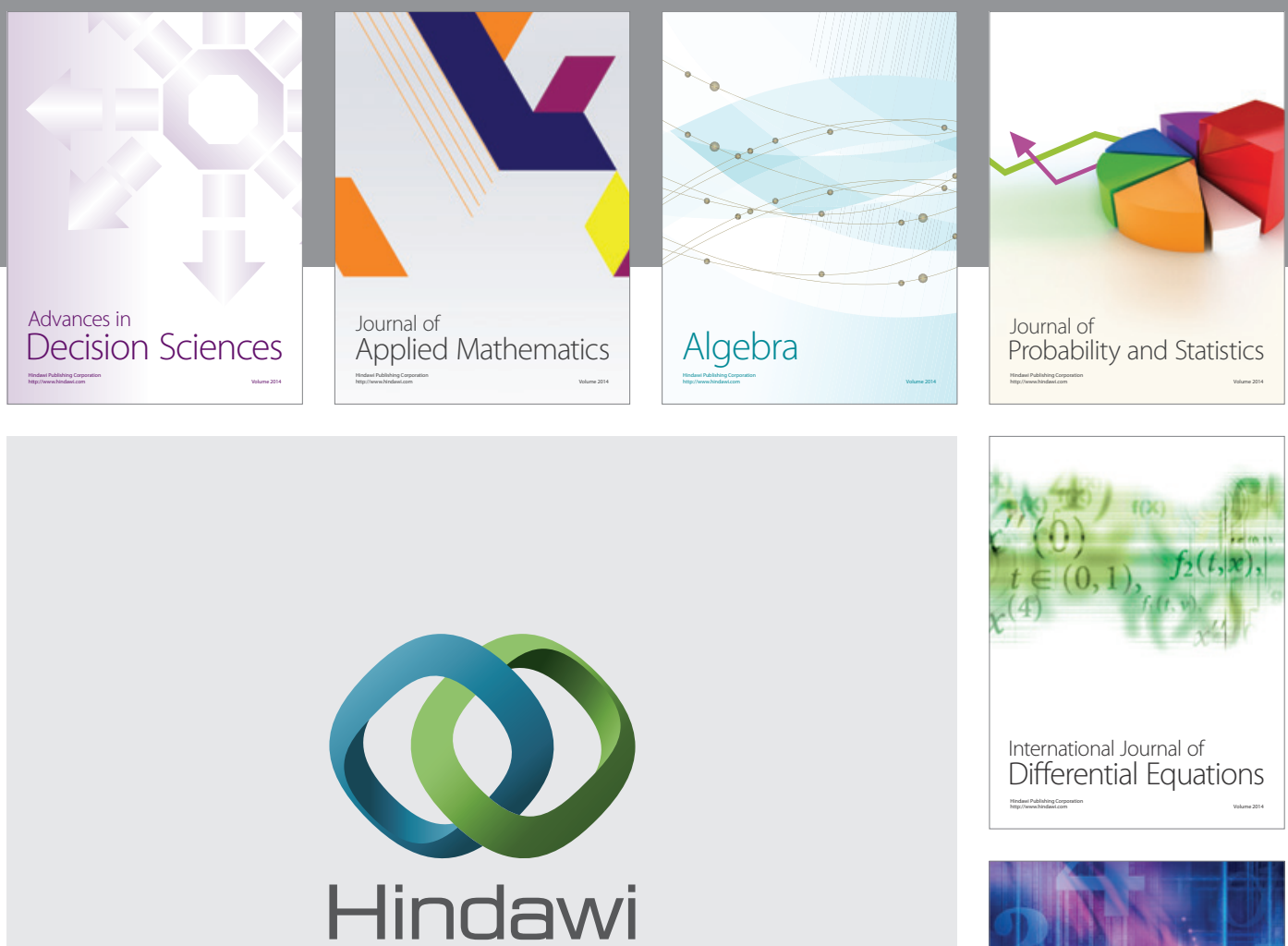

Submit your manuscripts at http://www.hindawi.com
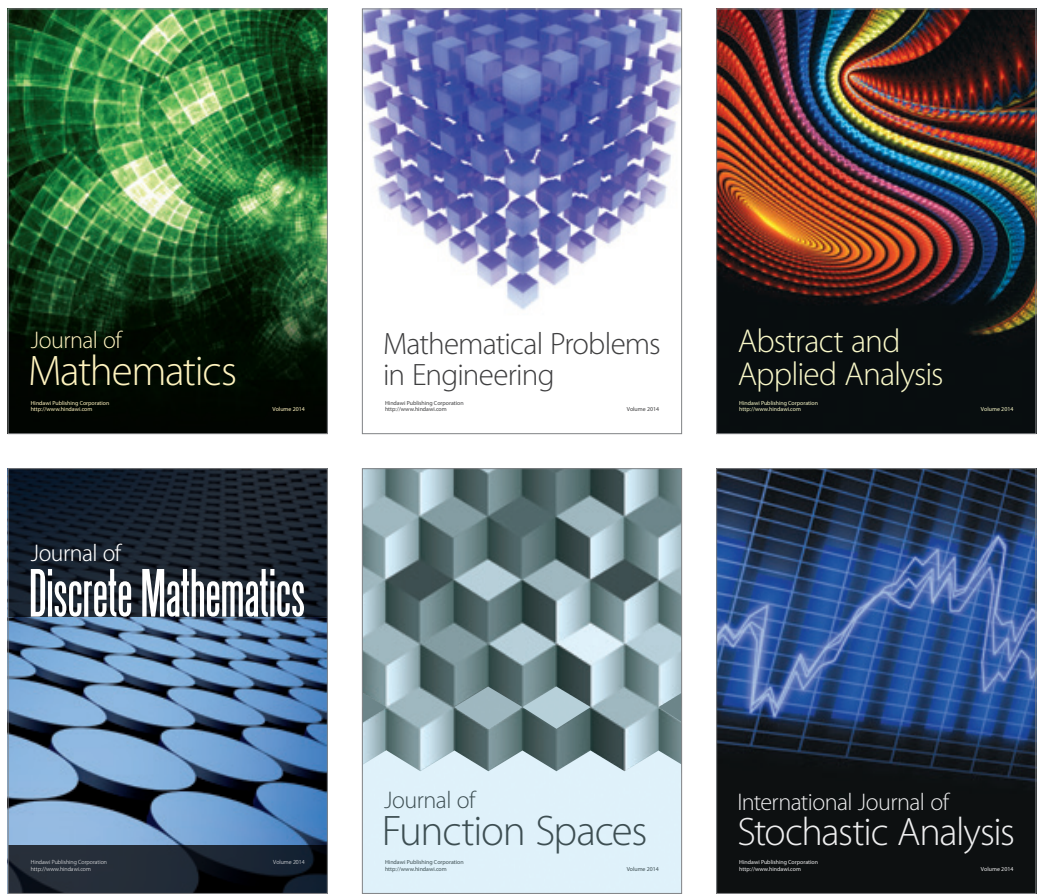

Journal of

Function Spaces

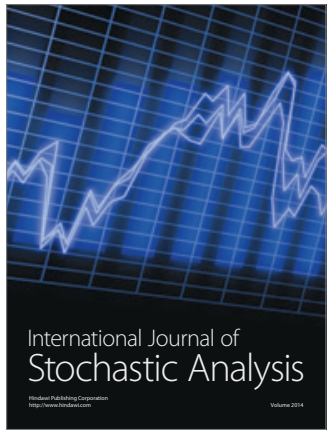

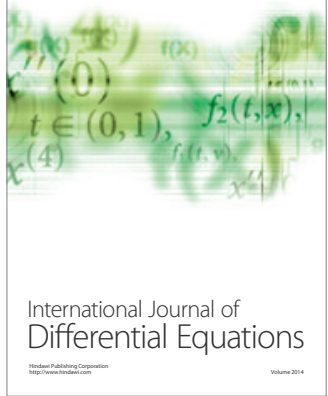
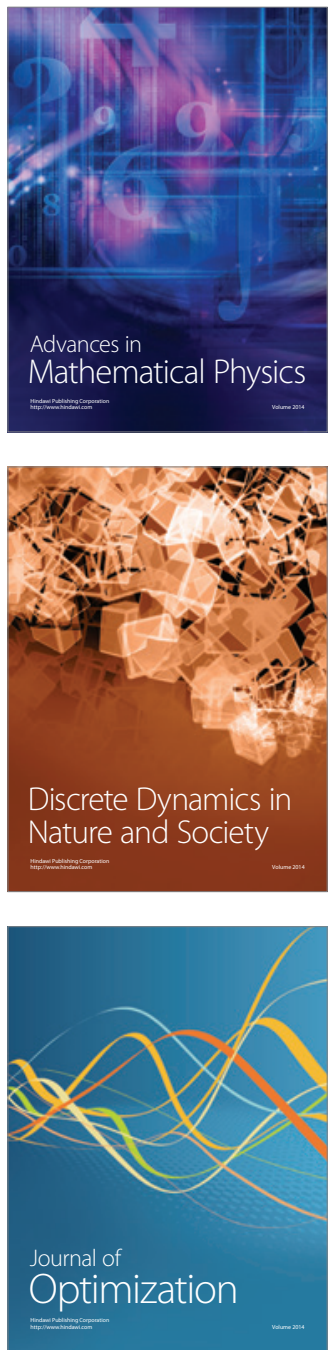